\title{
Endovascular Repair of Traumatic Common Iliac Vein Rupture
}

\author{
Chan Yong Park', Kwang Hee Yeo', Ho Hyun Kim¹, Jung Joo Hwang', \\ Seung Chan Lee', Chang Won Kim², Hyun Min Cho' \\ ${ }^{1}$ Department of Trauma Surgery, ${ }^{2}$ Department of Radiology, Pusan National University Hospital, Busan, Korea
}

\begin{abstract}
lliac vein rupture after blunt trauma is extremely rare, but there are some case reports describing endovascular repair of this lesion. However, there is no case report describing insertion of a stent graft in a patient with return of spontaneous circulation (ROSC) after cardiac arrest. Here we report our experience of performing stent graft insertion on a patient with ROSC with left common iliac vein rupture.
\end{abstract}

(Trauma Image Proced 2016(1):26-28)

Key Words: Iliac Vein; Wounds and Injuries; Endovascular Procedures; Stents

\section{CASE}

A 64-year-old male patient was transferred to our emergency department. He had experienced a return of spontaneous circulation (ROSC) after cardiopulmonary resuscitation (CPR) (performed owing to cardiac arrest) and was transferred to our hospital. On the abdominal computed tomography (CT) at the original hospital, a contrast leak was observed in the left common iliac vein. After arriving at our hospital, mechanical ventilation was initiated, and cardiac arrest and ROSC occurred twice more. After a massive transfusion, his blood pressure was $96 / 50 \mathrm{mmHg}$, and radiologic intervention was performed. Venography, which was done through puncture of the left common femoral vein, revealed rupture and massive extravasation (Fig. 2.). Through a $12 \mathrm{~F}$ sheath, a $16-12 \mathrm{~mm} / 100 \mathrm{~mm}$ stent graft (Gore Excluder stent graft) was inserted. Subsequent veno- graphy revealed disappearance of the extravasation (Fig. 3.). After the intervention, the patient's blood pressure recovered to $124 / 70 \mathrm{mmHg}$, and he underwent no further treatment in the intensive care unit. However, the status of the patient deteriorated, and he died the day after the intervention.

\section{DISCUSSION}

Blunt trauma-induced iliac vein rupture is rarely reported (1-3). Until now, there has been no case report describing stent insertion in an iliac vein in a patient with ROSC after CPR. Endovascular management of iliac vein rupture can be an option to consider for a patient whose hemodynamic status is relatively stable.

Received: June 2, 2016 Revised: July 11, 2016 Accepted: August 1, 2016

Correspondence to: Hyun Min Cho, Department of Trauma Surgery, Pusan National University Hospital, 179, Gudeok-ro, Seo-gu, Busan, Korea

Tel: 82-51-240-7369, Fax: 82-51-240-7719, E-mail: csking1@daum.net

Copyright (C) 2016 Korean Association for Research, Procedures and Education on Trauma. All rights reserved.

@) This is an open-access article distributed under the terms of the Creative Commons Attribution Non-Commercial License (http://creativecommons.org/ licenses/by-nc/4.0) which permits unrestricted noncommercial use, distribution, and reproduction in any medium, provided the original work is properly cited 


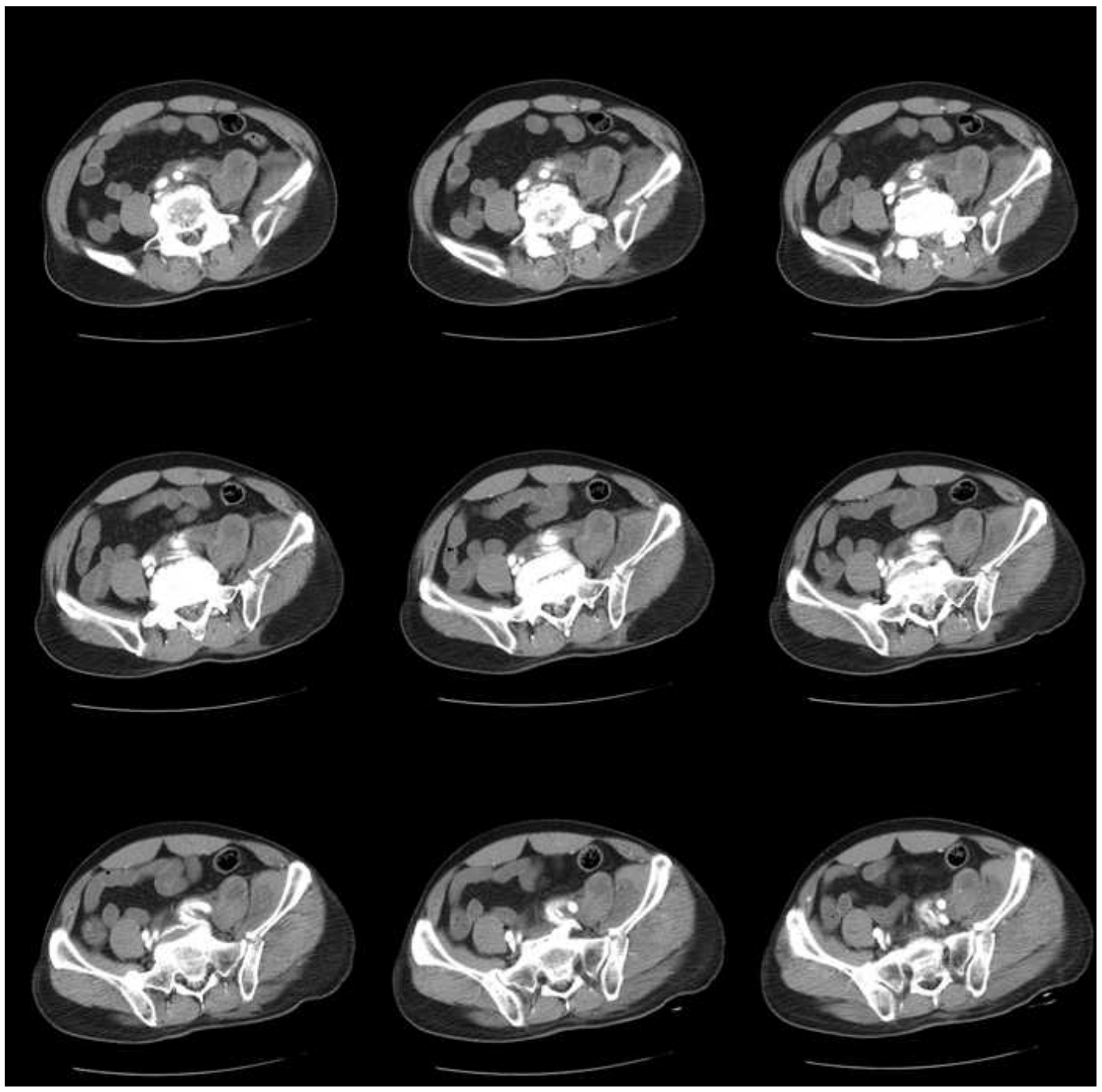

Fig. 1. Axial view of a computed tomography scan of the portal venous phase shows a left iliac bone fracture and contrast extravasation from the left common iliac vein.

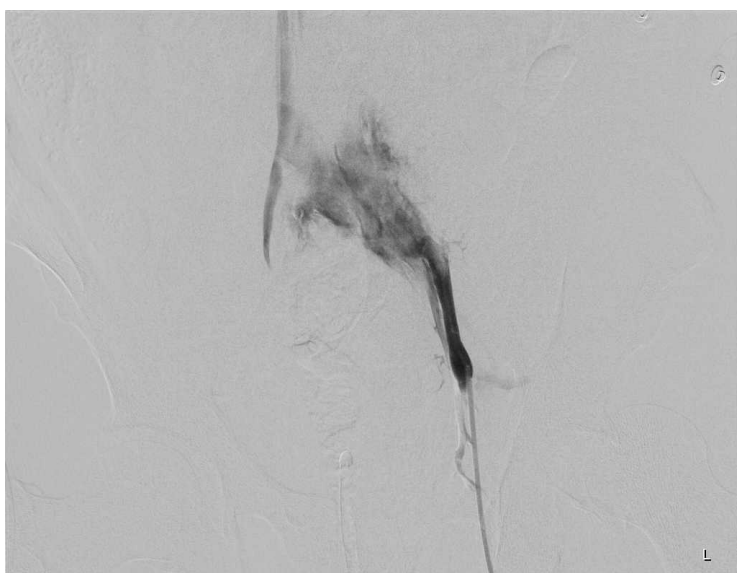

Fig. 2. Venography using left femoral access shows massive extravasation from the left common iliac vein. 


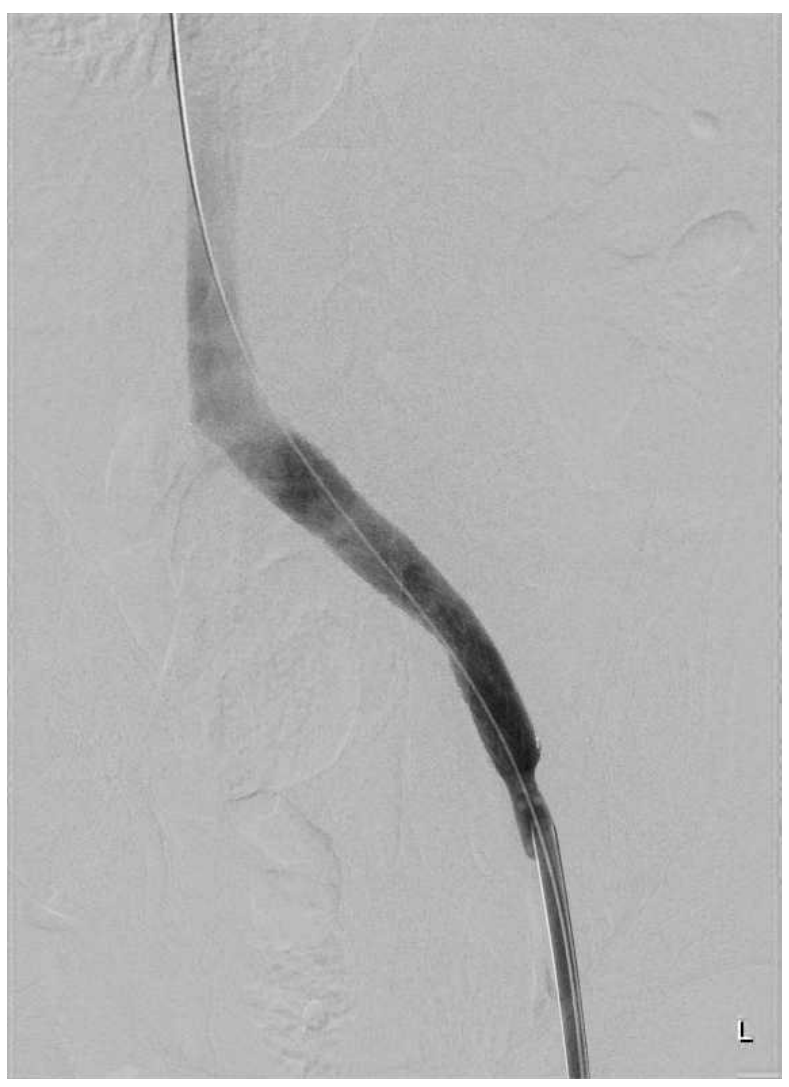

Fig. 3. Post-stent-graft venography shows disappearance of the contrast extravasation from the left common iliac vein.

\section{CONFLICT OF INTEREST}

No potential conflict of interest relevant to this article was reported.

\section{REFERENCES}

1. Boulanger B, Green J, Rodriguez A. Blunt traumatic iliac vein laceration without pelvic fracture: a rare entity. Journal of vascular surgery. 1992;16(1):87-9.

2. Buice WS, Hollenbeck JI, McElwee T. Isolated iliac vein injury from blunt trauma. Surgery. 1990;107(3):350-2.

3. Zieber SR, Mustert BR, Knox MF, Fedeson BC. Endovascular repair of spontaneous or traumatic iliac vein rupture. Journal of vascular and interventional radiology : JVIR. 2004;15(8):853-6. 